MITSUBISHI ELECTRIC RESEARCH LABORATORIES

http://www.merl.com

\title{
Global Optimization of Optimal Power Flow Using a Branch \& Bound Algorithm
}

\author{
Gopalakrishnan, A.; Raghunathan, A.U.; Nikovski, D.; Biegler, L.T.
}

TR2012-088 October 2012

\begin{abstract}
We propose two algorithms for the solution of the Optimal Power Flow (OPF) problem to global optimality. The algorithms are based on the spatial branch and bound framework with lower bounds on the optimal objective function value calculated by solving either the Lagrangian dual or the semidefinite programming (SDP) relaxation. We show that this approach can solve to global optimality the general form of the OPF problem including: generation power bounds, apparent and real power line limits, voltage limits and thermal loss limits. The approach makes no assumption on the topology or resistive connectivity of the network. This work also removes some of the restrictive assumptions of the SDP approaches [1], [2], [3], [4], [5]. We present the performance of the algorithms on a number of standard IEEE systems, which are known to have a zero duality gap. We also make parameter perturbations to the test cases that result in solutions that fail to satisfy the SDP rank condition and have a non-zero duality gap. The proposed branch and bound algorithms are able to solve these cases to global optimality.
\end{abstract}

Allerton Confernce on Communication, Control and Computing (Allerton)

\footnotetext{
This work may not be copied or reproduced in whole or in part for any commercial purpose. Permission to copy in whole or in part without payment of fee is granted for nonprofit educational and research purposes provided that all such whole or partial copies include the following: a notice that such copying is by permission of Mitsubishi Electric Research Laboratories, Inc.; an acknowledgment of the authors and individual contributions to the work; and all applicable portions of the copyright notice. Copying, reproduction, or republishing for any other purpose shall require a license with payment of fee to Mitsubishi Electric Research Laboratories, Inc. All rights reserved.
} 



\title{
Global Optimization of Optimal Power Flow Using a Branch \& Bound Algorithm
}

\author{
Ajit Gopalakrishnan* ${ }^{\dagger}$, Arvind U. Raghunathan ${ }^{\dagger}$, Daniel Nikovski ${ }^{\dagger}$ and Lorenz T. Biegler* \\ *Department of Chemical Engineering, Carnegie Mellon University, Pittsburgh, PA 15213. \\ Email: \{agopalak,lb01\}@andrew.cmu.edu \\ ${ }^{\dagger}$ Mitsubushi Electric Research Laboratories, Cambridge,MA 02139. \\ Email: \{raghunathan,nikovski\}@ merl.com
}

\begin{abstract}
We propose two algorithms for the solution of the Optimal Power Flow (OPF) problem to global optimality. The algorithms are based on the spatial branch and bound framework with lower bounds on the optimal objective function value calculated by solving either the Lagrangian dual or the semidefinite programming (SDP) relaxation. We show that this approach can solve to global optimality the general form of the OPF problem including: generation power bounds, apparent and real power line limits, voltage limits and thermal loss limits. The approach makes no assumption on the topology or resistive connectivity of the network. This work also removes some of the restrictive assumptions of the SDP approaches [1], [2], [3], [4], [5]. We present the performance of the algorithms on a number of standard IEEE systems, which are known to have a zero duality gap. We also make parameter perturbations to the test cases that result in solutions that fail to satisfy the SDP rank condition and have a non-zero duality gap. The proposed branch and bound algorithms are able to solve these cases to global optimality.
\end{abstract}

\section{INTRODUCTION}

Optimal Power Flow (OPF) problem for alternating current (AC) circuits concerns the problem of determining bus voltages and generator power levels to minimize a cost function. The cost functions employed include: generator cost, resistive losses or tertiary voltage control. The constraints for OPF include: (i) the AC power flow constraints, (ii) bounds on power generation, (iii) bounds on bus voltage magnitudes, (iv) bounds on thermal losses, and (v) limits on power transfer on lines. The OPF problem, first introduced by Carpentier [6], is a nonconvex optimization problem with quadratic constraints and quadratic objective function (QCQP). We provide a short survey of the existing literature and highlight some of the recent advances that have enabled the solution of OPF to global optimality. The assumptions associated with each of these methods are particularly emphasized.

\section{A. Literature Survery}

Initial attempts at solving the OPF considered simplifying the power flow equations using the DC-approximation [7], [8], [9]. This reduces the OPF to a convex Quadratic Program (QP). However, the assumption of small angle differences (DC-approximation) between the buses may not hold in general and this approximation turns out to perform poorly in the general case [10]. Nonlinear programming approaches have also been used to solve the OPF problem. A survey of the approaches for solving the OPF are provided in [11], [12]. For a more recent survey refer to [13].

Recently there has been an effort at obtaining globally optimal solutions to the nonconvex problems through second order cone programming (SOCP) and SDP relaxations. Jabr [14], [15] developed a SOCP relaxation for the load flow problem (generation levels fixed). The formulation dealt with variables in the polar form and was shown to be exact for tree networks. For meshed networks a successive approximation technique was proposed. Jabr subsequently extended the formulation to also address the OPF problem in [16] for tree networks. No results were provided for the case of meshed networks. The SDP approach for OPF was first considered by Bai and co-workers in [17], [18]. This idea was further analyzed by Lavaei and Low in [1] where the authors provided a sufficient condition (rank of solution $\leq 2$ ) for the SDP relaxation of the OPF to have zero duality gap. The authors empirically observed that a number of IEEE bus systems after perturbation of the resistances did satisfy the sufficiency condition. In an effort to provide necessary conditions, a number of subsequent papers [19], [4] have analyzed the conditions under which the rank condition can be guaranteed to be satisfied. The results have only been able to guarantee this for tree networks under the satisfaction of a technical condition which is difficult to guarantee. A drawback of the SDP approach is that there is no recourse to obtaining a feasible solution when the rank condition is not satisfied. This shortcoming was highlighted in the work of Lesieture et al. [20] who provided an example of a three bus system that violated the rank condition. There also exists an earlier work of Makarov et al. [21] that showed the nonconvexity of the power flow boundary.

Zhang and Tse [2] investigated conditions under which the boundary of the feasible injection region coincides with the convex hull of the feasible injection region. This provides another sufficient condition for OPF problems with objective functions that are strictly monotonic in the power injections. The authors showed that tree networks with voltage magnitude, real power line loss constraints, real power line flow constraints on every other line and bus power constraints at every other bus, have the same set of Pareto optimal points as its convex hull implying a zero duality gap. This result was also obtained subsequently using another approach by Bose et al. [4]. For non-tree networks, they obtain a weaker result 
by characterizing the convex hull of the voltage constraint injection region for lossless cycles, a lossless cycle with a chord and certain combinations of these networks.

For meshed networks, Sojoudi and Lavaei [5] consider a SOCP relaxation similar to that in [16]. They showed that the addition of sufficient number of phase shifters to edges in cycles can guarantee zero duality gap under certain assumptions such as load over-satisfaction and bounds only on power generation and voltage magnitudes.

Lavaei, Tse and Zhang [3] consider the relaxation of some of the restrictive assumptions in the work of Zhang and Tse [2] and Bose et al. [4]. The restrictions impose that the bounds can be allowed on power injections of alternate buses and real power line limits on alternate lines. They show that for practical tree networks, the OPF can be reduced to a convex problem when the voltage magnitudes at the buses are fixed and thermal lines losses, active line limits and bus limits are considered. The entire problem is cast in the space of angle differences between the buses with the power injection constraint taking the form of an ellipse while the rest of the constraints translate to linear inequalities. We stress again that all results are restricted to the case of tree networks.

All of the above cited work using SDPs utilized the bus injection formulation for the OPF. Farivar and Low [22] investigated the branch flow model for solving the OPF. They formulated the problem as a SOCP by relaxing some angle constraints. Their analysis requires that the objective function is a combination of line losses, linear costs on power generation and penalty on voltage magnitudes and also that only bounds on generation powers and bounds on voltage magnitudes are imposed. The formulation is shown to be exact for tree networks but for meshed networks they obtain a result similar to that of Sojoudi and Lavaei [5].

As mentioned earlier, the SDP approaches provide no recourse to obtaining a feasible solution when the rank condition is not satisfied. The work of Phan [23] takes the algorithmic route to achieving global optimality. The author proposes a spatial branch and bound approach with solutions of the Lagrangian dual providing lower bounds on the optimal objective function value. They consider the OPF problem with generation limits, voltage magnitude bounds and active line limits. Numerical experience showed that duality gap was closed at the root node without need for any branching on the IEEE test bus systems. These are the systems for which the SDP approach [1] also showed no duality gap.

\section{B. Our Contribution}

In this work, we are interested in solving to global optimality a given power network without making any restrictive assumptions on the topology of the network or constraints. We propose two Branch \& Bound (B \& B) algorithms, that rely on the SDP or Lagrangian dual relaxation to provide lower bounds on the globally optimal solution. The SDP based B \& B solves the SDP relaxation of [1] for lower bounding. The Lagrangian dual based B \& B algorithm builds on the work of [23], but is in the space of voltages and line power flows. This lifting into a higher dimensional space is necessary to incorporate the apparent power line flow constraints and to ensure that the Lagrangian dual can be solved efficiently. We compare the performance of the algorithm on several IEEE test cases. We then make small modifications to the parameters or constraints of the IEEE test cases for which the SDP formulation of [1] has a solution with rank greater than 2 . In some cases, even modifying the real and reactive power demands is sufficient to cause this violation. The proposed B \& B algorithms are able to provide an $\epsilon$-tolerance certificate of global optimality for these cases also.

The paper is organized as follows. Section II formulates the OPF problem and also its Lagrangian dual and SDP relaxations. Section II-C shows the equivalence of the Lagrangian dual and SDP relaxation for a general OPF problem. Section III outlines our SDP and Lagrangian dual based B \& B algorithm. Section IV provides the results and some directions for future work.

\section{Notation}

In the following, we use $\mathrm{j}$ to denote the imaginary root of -1 . For a complex variable $z, \operatorname{Re}(z)$ and $\operatorname{Im}(z)$ will denote the real and imaginary parts of the complex variable $z ;|z|$ will denote the magnitude of the complex variable and $z^{*}$ will denote the Hermitian conjugate. For a symmetric matrix $A$ its trace will be denoted as $\operatorname{Tr}(A)$ which is the sum of the diagonal entries.

\section{OPF PROBLEM}

We start with a given graph $\mathcal{G}=(\mathcal{N}, \mathcal{E})$ of the power network, where the nodes $i \in \mathcal{N}, j \in \mathcal{N}$ represent the buses and the edges in $\mathcal{E}$ represent branches connecting an ordered pair of buses $(i, j) . \mathcal{N}^{G} \subseteq \mathcal{N}$ denotes the set of buses connected to generators. Further we introduce $\mathcal{L}$ to denote the set in which the edges in $\mathcal{E}$ are duplicated, i.e. $(i, j) \in \mathcal{E} \Rightarrow(i, j),(j, i) \in \mathcal{L}$. For a bus $i, k \sim i$ denotes the set of buses $k$ which are connected to $i$. The line admittance is given by $y_{i j}=g_{i j}+\mathrm{j} b_{i j},(i, j) \in \mathcal{L}$, with $y_{i j}=0$ for $i \not j$. The bus admittance matrix, which relates the current injections to the bus voltages, is formed as

$$
Y_{\text {bus }}= \begin{cases}-y_{i j}, & i \neq j \\ y_{i i}+\sum_{k \sim i} y_{i k}, & i=j\end{cases}
$$

where $y_{i i}$ denotes the admittance-to-ground at bus $i$. Let $\mathbf{v}=$ $\left(V_{1}, V_{2}, \cdots, V_{|\mathcal{N}|}\right)$ denote the complex bus voltages, and $\mathbf{i}=$ $\left(I_{1}, I_{2}, \cdots, I_{|\mathcal{N}|}\right)$ denote the bus injection currents. Then by Ohm's and Kirchoff's laws applied on the entire network, $\mathbf{i}=$ $Y_{\text {bus }} \mathbf{v}$. Let $\mathbf{P}^{G}=\left(P_{1}^{G}, \cdots, P_{|\mathcal{N}|}^{G}\right), \mathbf{Q}^{G}=\left(Q_{1}^{G}, \cdots, Q_{|\mathcal{N}|}^{G}\right)$ denote the power generations at each bus, with $\left(P_{i}^{G}, Q_{i}^{G}\right)=$ $0, \forall i \notin \mathcal{N}^{G}$. The power flowing from bus $i$ to $j$ is calculated as,

$$
S_{i j}=V_{i}\left|V_{i}-V_{j}\right|^{*} y_{i j}^{*}
$$

The thermal loss on line $(i, j) \in \mathcal{E}$ is given by $L_{i j}=\mid V_{i}$ $\left.V_{j}\right|^{2} g_{i j}$. Note that $L_{i j}=\operatorname{Re}\left(S_{i j}\right)+\operatorname{Re}\left(S_{j i}\right)$. We define the 
voltages and branch power flows in the rectangular form,

$$
\begin{array}{rll}
V_{i} & :=e_{i}+\mathrm{j} f_{i} & \forall i \in \mathcal{N} \\
S_{i j} & :=P_{i j}+\mathrm{j} Q_{i j} & \forall(i, j) \in \mathcal{L}
\end{array}
$$

The classical OPF problem $(O P F)$ can be stated as,

$$
\begin{aligned}
& \min \sum_{i \in \mathcal{N}^{G}} f\left(P_{i}^{G}\right) \\
& \text { s.t. } P_{i}^{G}-P_{i}^{D}=\mathbf{x}^{T} Y_{i} \mathbf{x}, i \in \mathcal{N} \\
& Q_{i}^{G}-Q_{i}^{D}=\mathbf{x}^{T} \bar{Y}_{i} \mathbf{x}, i \in \mathcal{N} \\
& {\left[\alpha_{i}\right]} \\
& P_{i j}=\mathbf{x}^{T} Y_{i j} \mathbf{x},(i, j) \in \mathcal{L} \\
& {\left[\beta_{i}\right]} \\
& Q_{i j}=\mathbf{x}^{T} \bar{Y}_{i j} \mathbf{x},(i, j) \in \mathcal{L} \\
& {\left[\lambda_{i j}\right]} \\
& P_{i}^{\min } \leq P_{i}^{G} \leq P_{i}^{\max }, i \in \mathcal{N}^{G} \\
& {\left[\gamma_{i j}\right]} \\
& Q_{i}^{\min } \leq Q_{i}^{G} \leq Q_{i}^{\max }, i \in \mathcal{N}^{G} \\
& \left(V_{i}^{\min }\right)^{2} \leq \mathbf{x}^{T} M_{i} \mathbf{x} \leq\left(V_{i}^{\max }\right)^{2}, i \in \mathcal{N} \quad\left[\underline{\nu}_{i}, \bar{\nu}_{i}\right] \\
& P_{i j} \leq P_{i j}^{\max },(i, j) \in \mathcal{L} \\
& P_{i j}^{2}+Q_{i j}^{2} \leq\left(S_{i j}^{\max }\right)^{2},(i, j) \in \mathcal{L} \\
& \mathbf{x}^{T} M_{i j} \mathbf{x} \leq L_{i j}^{\max },(i, j) \in \mathcal{L} \\
& {\left[\mu_{i j}\right]}
\end{aligned}
$$

where $\mathbf{x}:=\left[\begin{array}{ll}\mathbf{e}^{T} & \mathbf{f}^{T}\end{array}\right]^{T}=\left[\operatorname{Re}(\mathbf{v})^{T} \operatorname{Im}(\mathbf{v})^{T}\right]^{T}$ and the matrices $Y_{i}, \bar{Y}_{i}, Y_{i j}, \bar{Y}_{i j}, M_{i}, M_{i j}$ are as defined in Appendix A. The objective function is to minimize the cost of generation, which is typically chosen to be convex quadratic, $f\left(P_{i}^{G}\right)=c_{2 i}\left(P_{i}^{G}\right)^{2}+c_{1 i} P_{i}^{G}+c_{0 i}$. Other objectives such as minimizing line losses could also be considered. Equations (2a), (2b) represent the real and reactive power balances at each bus. Equations (2c), (2d) are the real and reactive branch power flows, which are limited by constraints $(2 \mathrm{~h})$, (2i). Constraints (2e), (2f) are the power generation limits, $(2 \mathrm{~g})$ is the voltage magnitude limit and ( $2 \mathrm{j})$ is a limit on line losses. The dual multipliers corresponding to a subset of the constraints are shown in brackets [.].

It is well known that the OPF problem is nonconvex and we are interested in solving it to global optimality. We first outline two relaxations of the OPF based on the Lagrangian dual (LD) and semidefinite programming (SDP) and show their equivalence in Section II-C.

\section{A. Lagrangian Dual Relaxation of the $O P F$}

For brevity, we will denote by $\mathbf{z}:=\left(P^{G}, Q^{G}, P, Q\right)$, $\xi_{e q}:=(\alpha, \beta, \lambda, \gamma), \xi_{\text {in }}:=(\underline{\nu}, \bar{\nu}, \mu)$ and $\xi:=\left(\xi_{\text {eq }}, \xi_{\text {in }}\right)$. We dualize the model equations (2a)-(2d) with their corresponding multipliers in $\xi_{e q}$ and the the constraints $(2 \mathrm{~g})$, (2j) with their corresponding multipliers in $\xi_{\text {in }}$ to obtain the following Lagrangian function,

$$
\mathrm{L}(\mathbf{z}, \mathbf{x}, \xi):=\mathrm{L}^{1}(\mathbf{z}, \xi)+\mathbf{x}^{T} \mathbf{A}(\xi) \mathbf{x}
$$

where,

$$
\begin{aligned}
& \mathrm{L}^{1}(\mathbf{z}, \xi):=\sum_{i \in \mathcal{N}^{G}}\left(c_{2 i}\left(P_{i}^{G}\right)^{2}+c_{1 i} P_{i}^{G}+c_{0 i}\right) \\
& +\sum_{i \in \mathcal{N}^{G}}\left(\alpha_{i} P_{i}^{G}+\beta_{i} Q_{i}^{G}\right)+\sum_{(i, j) \in \mathcal{L}}\left(\lambda_{i j} P_{i j}+\gamma_{i j} Q_{i j}\right) \\
& +\sum_{i \in \mathcal{N}}\left(\underline{\nu}_{i}\left(V_{i}^{\min }\right)^{2}-\bar{\nu}_{i}\left(V_{i}^{\max }\right)^{2}\right)-\sum_{(i, j) \in \mathcal{L}} \mu_{i j} L_{i j}^{\max } \\
& -\sum_{i \in \mathcal{N}}\left(\alpha_{i} P_{i}^{D}+\beta_{i} Q_{i}^{D}\right)
\end{aligned}
$$

and

$$
\begin{aligned}
& \mathbf{A}(\xi):=\sum_{i \in \mathcal{N}}\left(-\alpha_{i} Y_{i}-\beta_{i} \bar{Y}_{i}+\left(\bar{\nu}_{i}-\underline{\nu}_{i}\right) M_{i}\right) \\
& +\sum_{(i, j) \in \mathcal{L}}\left(-\lambda_{i j} Y_{i j}-\gamma_{i j} \bar{Y}_{i j}+\mu_{i j} M_{i j}\right)
\end{aligned}
$$

The Lagrangian dual function $g(\xi)$ can be written as,

$$
g(\xi):=\inf _{\mathbf{z}, \mathbf{x}} \mathrm{L}(\mathbf{z}, \mathbf{x}, \xi) \text { s.t. } \quad(2 \mathrm{e}),(2 \mathrm{f}),(2 \mathrm{~h}),(2 \mathrm{i})
$$

and the optimal value of the dual function is denoted by,

$$
\max _{\xi} g(\xi) \text { s.t. } \quad \xi_{\text {in }} \geq 0, \xi_{e q} \text { free. }
$$

Constraints (2e), (2f), (2h) and (2i) are not dualized as they are easy to handle. We defer details on the solution of the Lagrangian dual to Section III-A.

\section{B. SDP Relaxation of the OPF}

We introduce the following convex formulation with a lifting of the quadratic voltage terms $\mathrm{xx}^{\mathrm{T}}$ to a semidefinite matrix while retaining the remaining variables. This is done so as to only relax the variables that appear in a nonconvex fashion in the constraints. Denoting by $\mathbf{W}$ the $2|\mathcal{N}| \times 2|\mathcal{N}|$ symmetric matrix and dropping the $\operatorname{rank}(\mathbf{W})=1$ condition, we formulate the SDP relaxation as follows,

$$
\begin{array}{ll}
\min & \sum_{i \in \mathcal{N}^{G}}\left(c_{2 i}\left(P_{i}^{G}\right)^{2}+c_{1 i} P_{i}^{G}+c_{0 i}\right) \\
\text { s.t. } & (2 \mathrm{e}),(2 \mathrm{f}),(2 \mathrm{~h}),(2 \mathrm{i}) \\
& P_{i}^{G}-P_{i}^{D}=\operatorname{Tr}\left(Y_{i} \mathbf{W}\right), Q_{i}^{G}-Q_{i}^{D}=\operatorname{Tr}\left(\bar{Y}_{i} \mathbf{W}\right) \\
& P_{i j}=\operatorname{Tr}\left(Y_{i j} \mathbf{W}\right), Q_{i j}=\operatorname{Tr}\left(\bar{Y}_{i j} \mathbf{W}\right) \\
& \left(V_{i}^{\min }\right)^{2} \leq \operatorname{Tr}\left(M_{i} \mathbf{W}\right) \leq\left(V_{i}^{\max }\right)^{2} \\
& \operatorname{Tr}\left(M_{i j} \mathbf{W}\right) \leq L_{i j}^{\max } \\
& \mathbf{W} \succeq 0 .
\end{array}
$$

The dual of the SDP relaxation can then be written in a manner analogous to the previous section,

$$
\mathrm{L}^{\mathrm{SDP}}(\mathbf{z}, \mathbf{W}, \xi):=\mathrm{L}^{1}(\mathbf{z}, \xi)+\operatorname{Tr}(\mathbf{A}(\xi), \mathbf{W}) .
$$

The Lagrangian dual function is given by

$$
\begin{aligned}
g^{\mathrm{SDP}}(\xi):= & \inf _{\mathbf{z}, \mathbf{W}} \mathrm{L}^{\mathrm{SDP}}(\mathbf{z}, \mathbf{W}, \xi) \\
& \text { s.t. }(2 \mathrm{e}),(2 \mathrm{f}),(2 \mathrm{~h}),(2 \mathrm{i}), \mathbf{W} \succeq 0 .
\end{aligned}
$$

and the optimal value of the dual function is denoted by,

$$
\max _{\xi} g^{\mathrm{SDP}}(\xi) \text { s.t. } \quad \xi_{i n} \geq 0, \xi_{\text {eq }} \text { free. }
$$

\section{Equivalence}

To establish the equivalence between the Lagrangian dual (4) and SDP relaxation (5), first note that if $O P F$ (2) is feasible then so is its SDP relaxation (5). Hence, strong duality holds for SDP (5) and its dual (7) under Slater's condition. Now, consider the Lagrangian dual function of the OPF $g(\xi)$, and of the SDP relaxation $g^{\mathrm{SDP}}(\xi)$. Firstly, observe that the optimization problem defining $g(\xi)$ is separable in $\mathbf{z}$ and $\mathbf{x}$. Similarly, the optimization problem defining $g^{\mathrm{SDP}}(\xi)$ is also separable in $\mathbf{z}$ and $\mathbf{W}$. Further, the objective functions and constraints with respect to $\mathbf{z}$ are identical in (3) and (6). It 
only remains to resolve the contribution in the dual functions due to $\mathbf{x}$ in (3) and due to $\mathbf{W}$ in (6). For this consider the conditions under which $\inf _{\mathbf{x}} \mathbf{x}^{T} \mathbf{A}(\xi) \mathbf{x}$ is finite-valued. This occurs only when $\mathbf{A}(\xi) \succeq 0$. It is easily seen that the same condition is required for the term $\inf _{W \succeq 0} \operatorname{Tr}(\mathbf{A}(\xi), \mathbf{W})$ to be finite valued. This establishes that the Lagrangian dual of the OPF (4) is equivalent to the dual of the SDP relaxation (7). The equivalence is far more general and can be shown to hold for any QCQP [24].

The optimal value of the Lagrangian dual yields a lower bound on the original problem due to weak duality for the nonconvex $O P F$. The optimal value of the SDP relaxation likewise yields a lower bound on the original problem, even if the rank $\leq 2$ condition fails. So we have,

$$
\mathrm{LD}=\mathrm{SDP}=\text { dual } \mathrm{SDP} \leq O P F(2)
$$

and the optimal Lagrangian multipliers corresponding to the Lagrangian dual are nothing but the optimal dual vector for the SDP. However, computationally they are not equivalent. Finding the optimal Lagrangian multipliers might be a challenge in practice as the Lagrangian dual of the OPF is non-smooth, and the convergence behavior of nonsmooth algorithms tend to be slower compared to their smooth counterparts [25]. On the other hand, the SDP relaxation is a smooth problem that can be solved in polynomial time by interior point algorithms [26].

\section{GlobAl OptimizATION BY BRANCH AND BOUND}

The branch and bound method is a general purpose global optimization technique for a wide class of nonconvex problems. It solves the problem $\mathrm{P}$ by constructing a convex relaxation $\mathrm{R}$, that is easy to solve and provides a lower bound $(L)$ on the optimal objective function value (Figure 1a). The upper bound $(U)$ can be arrived at by using local minimization, which also yields a feasible solution. If $U-L$ is sufficiently small, the procedure terminates with the current upper bounding solution. Otherwise, the feasible region is recursively partitioned, and the procedure is repeated (Figure 1b) until the gap $U-L$ is sufficiently small. Nodes are fathomed if the lower bound $L$ is greater than the current best upper bound (Figure 1c). We refer the interested reader to [27] for additional information.

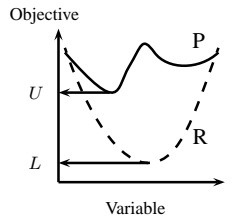

(a) Lower and Upper Bounding

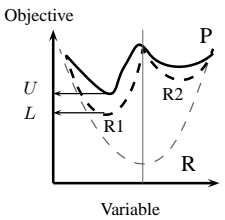

sion

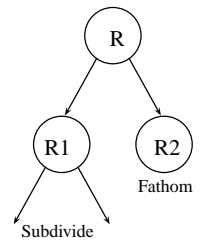

(c) Search tree
Fig. 1: Branch and Bound Schematic

In this work, the upper bounding problem is done through CONOPT [28], a local nonlinear programming (NLP) solver. The lower bounding is done through either the SDP relaxation or the Lagrangian dual, both of which are equivalent but could differ in computational performance. If there is an optimality gap, the feasible region is partitioned into two sub-regions, over which the procedure is repeated. The partitioning is done as follows. At the root node 0 of the branch and bound tree, define

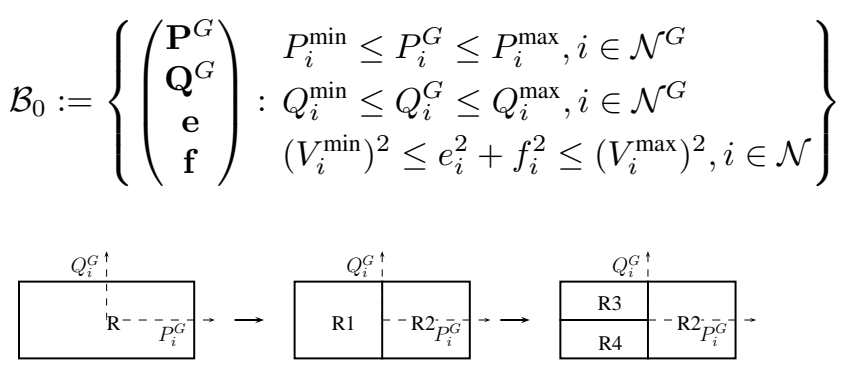

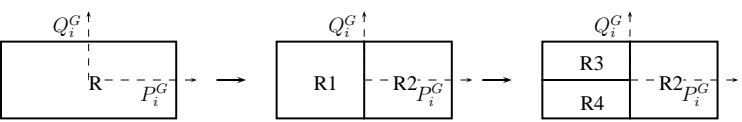

(a) Rectangular Bisections

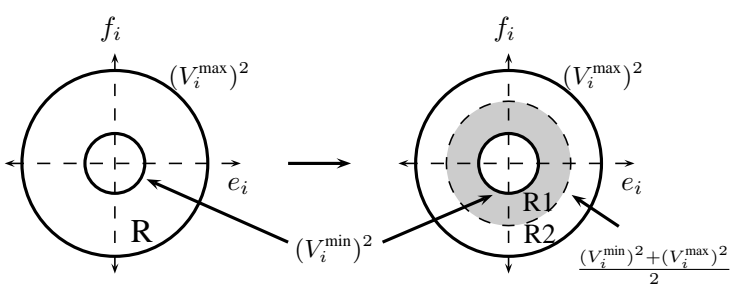

(b) Radial bisections

Fig. 2: Domain subdivision in the B \& B algorithm.

Subproblems R1 and R2 are created by either rectangular bisection on $P_{i}^{G}$ or $Q_{i}^{G}$, or by radial bisection on the voltage magnitudes $\left(e_{i}^{2}+f_{i}^{2}\right)$ as shown in Figure 2. The newly generated bounds $\overline{\mathcal{B}}$,

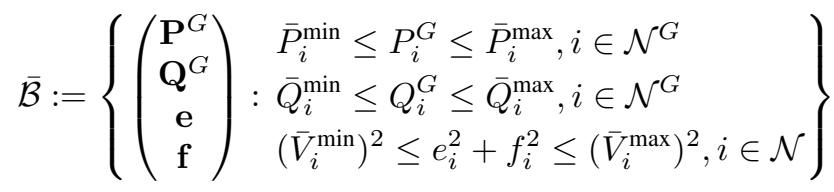

replace the existing bounds in the upper bounding problem $O P F$ and in the Lagrangian dual \& SDP relaxations.

\section{A. Lagrangian dual based branch and bound}

The minimization of the Lagrangian in (3) is tractable because it can be decomposed into individual problems for the generators, line flows and voltages as follows. The decomposition for the generator and voltage subproblems follows along the lines of [23]. The line flow subproblem however represents an extension of the work in [23].

Generator subproblems: $\left(L D_{i}^{1}\right) \quad \forall i \in \mathcal{N}^{G}$

$$
\begin{aligned}
\min _{P_{i}^{G}, Q_{i}^{G}} & \sum_{i \in \mathcal{N}^{G}}\left[c_{2 i}\left(P_{i}^{G}\right)^{2}+c_{1 i} P_{i}^{G}+c_{0 i}+\alpha_{i} P_{i}^{G}+\beta_{i} Q_{i}^{G}\right] \\
\text { s.t. } & P_{i}^{\min } \leq P_{i}^{G} \leq P_{i}^{\max }, \forall i \in \mathcal{N}^{G} \\
& Q_{i}^{\min } \leq Q_{i}^{G} \leq Q_{i}^{\max }, \forall i \in \mathcal{N}^{G}
\end{aligned}
$$

It is easy to see (8) is convex and can be solved analytically for each generation variable.

Line flow subproblems: $\left(L D_{i j}^{2}\right) \quad \forall(i, j) \in \mathcal{L}$

$$
\begin{aligned}
\min _{P_{i j}, Q_{i j}} & \lambda_{i j} P_{i j}+\gamma_{i j} Q_{i j} \\
\text { s.t. } & P_{i j} \leq P_{i j}^{\max },\left(P_{i j}\right)^{2}+\left(Q_{i j}\right)^{2} \leq\left(S_{i j}^{\max }\right)^{2}
\end{aligned}
$$


The $|\mathcal{L}|$ line flow subproblems are also convex, decoupled and can be solved analytically for each line flow variable. Voltage subproblem: $\left(L D^{3}\right)$

$$
\begin{array}{cl}
\min _{\mathbf{x}} & \mathbf{x}^{T} \mathbf{A}(\xi) \mathbf{x} \\
\text { s.t. } & \Delta_{L} \leq \mathbf{x}^{T} \mathbf{x} \leq \Delta_{U}
\end{array}
$$

where $\Delta_{L}=\sum_{i \in \mathcal{N}}\left(V_{i}^{\min }\right)^{2}, \Delta_{U}=\sum_{i \in \mathcal{N}}\left(V_{i}^{\max }\right)^{2}$. Two redundant spherical inequalities are added in order to tighten the bounds obtained from solving the Lagrangian dual as in [23]. The voltage subproblem is nonconvex and we need to find its global minimum. This is a special case of the trust-region problem: minimization of a purely quadratic objective subject to two ball constraints, the global solution of which is simply given by the eigenvector corresponding to the mimimum eigenvalue of the quadratic term.

$$
\mathbf{x}^{\text {opt }}= \begin{cases}\mathbf{x}^{\min } \frac{\Delta_{U}}{\left\|\mathbf{x}^{\min }\right\|}, & \text { if } e^{\min }<0 \\ \mathbf{x}^{\min } \frac{\Delta_{L}}{\left\|\mathbf{x}^{\min }\right\|}, & \text { if } e^{\min } \geq 0\end{cases}
$$

where $\mathrm{x}^{\mathrm{min}}$ is the eigenvector corresponding to the smallest eigenvalue $e^{\mathrm{min}}$ of $\mathbf{A}(\xi)$. For large and sparse matrices, the smallest eigenvalue is fairly easy to compute through a Krylov subspace method involving matrix multiplications only. The Matlab function eigs implements this.

The Lagrangian dual provides a lower bound on the optimal value of $O P F(2)$, and in order to get the best lower bound we have to solve (4). This is a non-smooth concave maximization problem, which can be solved using a projected subgradient method. It involves a simple iterative update of the form,

$$
\xi_{e q}^{(k+1)}=\xi_{e q}^{(k)}+\alpha^{(k)} \mathbf{g}_{e q}^{(k)}, \quad \xi_{i n}^{(k+1)}=\left[\xi_{i n}^{(k)}+\alpha^{(k)} \mathbf{g}_{i n}^{(k)}\right]^{+}
$$

There are several ways to choose the stepsize $\alpha^{(k)}$ and search direction $\mathbf{g}^{(k)}$. The method in [29] suggests choosing the step size as $\alpha^{(k)}=\left(q^{(k)}-\hat{q}^{*}\right) /\left\|\mathbf{g}^{(k)}\right\|_{2}^{2}$, where $\hat{q}^{*}$ is an estimate on the best dual value which can be set as $z_{\text {live }}^{*}$ (refer algorithm). The search direction $\mathbf{g}^{(k)}$ is computed through a conic combination of the subgradient and the direction at the previous iteration,

$$
\mathbf{g}^{(k)}=\mathbf{d}^{(k)}+\beta^{(k)} \mathbf{g}^{(k-1)}
$$

with $\beta^{(k)}=\max \left(0,-1.5\left(\mathbf{g}^{(k-1)}\right)^{T} \mathbf{d}^{(k)} /\left\|\mathbf{g}^{(k-1)}\right\|_{2}^{2}\right)$. One element of the subgradient $\mathbf{d}^{(k)}$ is simply the residual of the constraint corresponding to its multiplier in the Lagrangian, $\mathbf{d}^{(k)}=\left(\frac{\partial \mathrm{L}}{\partial \xi}\right)^{(k)}$.

The subgradient method does not guarantee ascent, and it can take a large number of iterations before the optimal multipliers $\xi$ are found. Hence it is usually terminated after a fixed number of iterations (maxiter). We now state the branch and bound algorithm using the Lagrangian dual.

ALGORITHM for LD-BB

1. Set BestUB $=$ inf, BestlB $=-$ inf, nodes $=\{0\}$.

2. If (nodes $\neq \emptyset$ and (BestUB - BestLB)/BestUB $>$ tol), choose a live node to explore from nodes.
Else return best upper bounding solution as global optimum and STOP.

3. Solve $O P F(2)$ using an NLP solver with bounds $\mathcal{B}_{\text {live }}$

(a) If $O P F$ is infeasible, fathom 1 ive. Go to Step 5.

(b) If $O P F$ is feasible with objective $z_{\text {live }}^{*}$, update BestUB $=\min \left(\right.$ BestUB, $\left.z_{\text {live }}^{*}\right)$.

4. Solve $L D$ (4) using a subgradient method with bounds $\mathcal{B}_{\text {live. }}$

(a) If at any iteration $k \leq$ maxiter of the subgradient method, $q^{(k)}>$ BestUB or (BestUB $\left.q^{(k)}\right) /$ BestUB $\leq$ tol, fathom live. Go to Step 5 .

(b) At termination of subgradient method, set $q_{\text {live }}^{*}=$ $\max _{k} q^{(k)}$. Partition $\mathcal{B}_{\text {live }}$ into two regions and update nodes $=\{$ nodes, child1, child2 $\}$. $\operatorname{LB}(\operatorname{child} 1)=\operatorname{LB}(\operatorname{child} 2)=q_{\text {live }}^{*}$. Remove live from nodes.

5. Set BestLB $=\min (\operatorname{LB}($ nodes $))$. Go to Step 2 .

In Step 3(a) we rely on the robustness of the NLP solver to detect infeasibilities. This assumption was not too restrictive for the problems we considered and helped to accelerate convergence.

Speeding up the subgradient method: Though subgradient methods are presumed to be slow in practice, we were able to speed it up through two observations.

- We have a tight bound on the optimal value $\hat{q}^{*}$ of the Lagrangian dual from the solution of the NLP at each node of the branch and bound tree. A stepsize selection based on $\hat{q}^{*}$ is known to considerably speed up the method [25]. This also helped in fathoming nodes early in Step 4(a) of the algorithm.

- The dual variables corresponding to the NLP optimal solution provide a very good initial point as they are a valid lower bound on the dual objective. A better initial point is also available from the best dual solution of its parent node in the branch and bound tree. This warm start technique helps to improve the performance of the algorithm at the expense of increased storage.

\section{B. SDP based branch and bound}

We now state the SDP based branch and bound algorithm. The SDP relaxation at each node is solved using SeDuMi through the YALMIP interface [30] in Matlab. Instead of solving the dual of (5), we solve the dual of the SDP relaxation as suggested by [1], i.e. in the space of only voltages and with all constraints dualized.

ALGORITHM for SDP-BB

1. Set BestUB $=$ inf, BestlB $=-$ inf, nodes $=\{0\}$.

2. If (nodes $\neq \emptyset$ and (BestUB - BestLB)/BestUB $>$ tol), choose a live node to explore from nodes. Else return best upper bounding solution as global optimum and STOP.

3. Solve $O P F(2)$ using an NLP solver with bounds $\mathcal{B}_{\text {live }}$

(a) If $O P F$ is infeasible, fathom live. Go to Step 5.

(b) If $O P F$ is feasible with objective $z_{\text {live, }}^{*}$ update BestUB $=\min \left(\right.$ BestUB, $\left.z_{\text {live }}^{*}\right)$. 
4. Solve $S D P$ (7) using SeDuMi with bounds $\mathcal{B}_{\text {live }}$.

(a) If $S D P$ is infeasible, fathom live. Go to Step 5.

(b) If $S D P$ is feasible with objective $q_{1 \text { ive }}^{*}$

(i) If $q_{\text {live }}^{*}>$ BestuB or (BestuB $\left.q_{\text {live }}^{*}\right) /$ BestUB $\leq$ tol, fathom live. Go to Step 5.

(ii) Partition $\mathcal{B}_{\text {live }}$ into two regions and update nodes $=$ nodes, child1, child2 . $\operatorname{LB}(\operatorname{child} 1)=\operatorname{LB}(\operatorname{child} 2)=q_{\text {live }}^{*}$. BestLB $=\min (\mathrm{LB}($ nodes $)) . \quad$ Remove live from nodes.

5. Set BestlB $=\min (\mathrm{LB}($ nodes $))$. Go to Step 2 .

\section{Convergence to Global Optimum}

We provide a proof of convergence for the algorithms.

Remark 1. Since the subdivision of the bounds $\mathcal{B}$ involves a bisection of $\mathbf{P}^{G}$, the feasible region for $\mathbf{P}^{G}$ shrinks at every step. If the resulting OPF problem is feasible then, so is the $S D P$ relaxation in (5). Further, the upper and lower bounds also converge in the limit as the objective function value is a function of only $\mathbf{P}^{G}$. The bounding scheme is thus consistent [27] (p. 6). From the equivalence of the SDP relaxation and Lagrangian dual we have that the algorithm based on the latter method is also consistent.

Remark 2. The bisections on $\mathbf{Q}^{G}$ and $(\mathbf{e}, \mathbf{f})$ help in raising the lower bound and speeding up convergence.

Remark 3. The selection of the live node to be explored in Step 2 is done by picking the one with the best lower bound. This ensures the algorithm is bound improving [27] (p. 6).

Theorem 1. The B\&B algorithms based on Lagrangian Dual and SDP relaxation converge to the globally optimal solution.

Proof: The branch and bound algorithm we have proposed is both consistent and bound improving. Hence, it is also convergent [27] (p. 6).

\section{Results}

In this section we report the results from the branch and bound algorithms applied on multiple test cases. The algorithms were implemented in Matlab 7.11 and executed on a machine with Intel Core i7 $3.4 \mathrm{GHz}$ running under Windows 7 operating system. All computations were executed on a single processor. We first solve the following test cases,

- 3bus example from [20]

- Contour 6bus example from PowerWorld [31]

- caseww6, case9 from the MATPOWER data set [32]

- New England 39-bus case

- IEEE14, IEEE30, IEEE57, IEEE118 test cases.

Table I shows the results from the two algorithms $L D-B B$ and $\mathrm{SDP}-\mathrm{BB}$ on the test cases. In addition, we also make a comparison with BARON [33], a powerful general purpose global optimization solver available under GAMS [34]. BARON uses linear programming relaxations to solve the lower bounding problem.
For both the algorithms we have proposed, the optimality gap was closed at the root node itself, corroborating the findings of [23]. The optimal solution for caseww6 and NE39 examples is slightly different from [23] due to the inclusion of apparent line limit constraints. We would also like to point out that the small perturbation to the resistance term $\left(10^{-5}\right.$ p.u.) suggested by [1] is not required here as the SDP solution is only used to provide a lower bound. The CPU times for both the branch and bound algorithms are of the order of seconds. The maximum time limit (600 CPU sec) was reached by BARON (with default options) in most of the test cases, indicating a weakness of the linear relaxations used by the solver to compute the lower bound.

\section{A. Perturbed cases}

In all of the above test cases, the SDP and Lagrangian dual relaxations were able to close the gap at the root node. However, in the presence of line flow constraints [20] or lower bounds on reactive power generations [2], the SDP approach is not guaranteed to give a rank 2 solution and consequently we expect a non-zero duality gap at the root node. We modify the following operational parameters in some of the test cases: apparent line power flow limits, real and reactive power demands, and lower bounds on reactive power generation, in order to simulate case studies with a non-zero duality gap. The modifications are shown in Table II. In some cases, we found that just a change in real and reactive power demands was sufficient to cause a violation of the rank 2 condition. None of the physical parameters of the network were modified.

\begin{tabular}{|c|l|l|c|}
\hline$\#$ & Test bus & Changes & SDP Rank \\
\hline 1. & 3bus & $S_{3}^{\max }=50$ & 4 \\
2. & IEEE9 & $\mathbf{Q}^{\min }=10, \mathbf{P}^{D}=1.1 \mathbf{P}^{D}$ & 6 \\
3. & IEEE14 & $\mathbf{Q}^{\min }=0, \mathbf{Q}^{D}=0.1 \mathbf{Q}^{D}$ & 6 \\
4. & IEEE30 & $\mathbf{P}^{D}=0.5 \mathbf{P}^{D}, \mathbf{Q}^{D}=0.1 \mathbf{Q}^{D}$ & 10 \\
5. & IEEE57 & $\mathbf{P}^{D}=1.06 \mathbf{P}^{D}, \mathbf{Q}^{D}=1.06 \mathbf{Q}^{D}$ & 4 \\
\hline
\end{tabular}

TABLE II: Modified test cases

Though the SDP and Lagrangian dual relaxations give the same theoretical bounds, it remains to be seen which one is the more effective approach. The LD (4) is a concave maximization problem whose optimal value is the same as SDP (7), but we may not be able to reach that value within a finite number of iterations due to computational difficulties associated with the non-smooth nature of the Lagrangian dual. We set a stopping criteria of maxiter $=500$ for the subgradient method. The convergence criterion for both algorithms was set at relative gap of tol $=0.01$ (Table III) and tol $=0.001$ (Table IV). This corresponds to converging the quantity BestUB - BestLB to within $1 \%$ and $0.1 \%$ respectively of BestUB. The root node gap and gap at termination of the algorithms, the wall clock time and \# nodes opened in the B \& B tree are also shown.

The SDP-BB algorithm was able to arrive at and certify the globally optimal solution to a $1 \%$ tolerance at the root node itself for all the cases. The LD-BB algorithm was able to do 


\begin{tabular}{|l|l|l|l|l|l|l|l|l|}
\hline \multicolumn{2}{|c|}{} & \multicolumn{3}{c|}{ SDP-BB } & \multicolumn{3}{c|}{ LD-BB } & BARON \\
\hline Case & BestUB & gap \% & Time (sec) & Nodes & gap \% & Time (sec) & Nodes & Time (sec) \\
\hline 3bus & 5707.10 & 0 & 2.83 & 1 & 0 & 0.32 & 1 & 0.58 \\
6bus & 7381.82 & 0 & 2.15 & 1 & 0 & 0.33 & 1 & $1 \mathrm{im}$ \\
caseww6 & 3143.97 & 0 & 2.23 & 1 & 0 & 0.30 & 1 & 3.01 \\
IEEE9 & 5296.69 & 0 & 2.27 & 1 & 0 & 0.32 & 1 & $1 \mathrm{im}$ \\
IEEE14 & 8081.53 & 0 & 2.42 & 1 & 0 & 0.33 & 1 & $1 \mathrm{im}$ \\
IEEE30 & 8906.14 & 0 & 3.38 & 1 & 0 & 0.39 & 1 & $1 \mathrm{im}$ \\
NE39 & 41862.10 & $<0.1$ & 4.73 & 1 & $<0.1$ & 6.20 & 1 & $1 \mathrm{im}$ \\
IEEE57 & 41737.79 & $<0.1$ & 7.63 & 1 & $<0.1$ & 0.85 & 1 & $1 \mathrm{im}$ \\
IEEE118 & 129660.68 & $<0.1$ & 28.52 & 1 & $<0.1$ & 3.75 & 1 & $1 \mathrm{im}$ \\
\hline
\end{tabular}

TABLE I: Branch and bound algorithm solved to a relative gap (tol) of $0.1 \%$.

\begin{tabular}{|c|r|c|c|l|r|r|l|l|}
\hline & \multicolumn{3}{|c|}{ SDP-BB } & \multicolumn{4}{|c|}{ LD-BB } \\
\hline$\#$ & $\begin{array}{r}\text { root } \\
\text { gap\% }\end{array}$ & $\begin{array}{l}\text { closed } \\
\text { gap\% }\end{array}$ & $\begin{array}{r}\text { Time } \\
(\mathrm{sec})\end{array}$ & Nodes & $\begin{array}{r}\text { root } \\
\text { gap\% }\end{array}$ & $\begin{array}{l}\text { closed } \\
\text { gap\% }\end{array}$ & $\begin{array}{r}\text { Time } \\
(\mathrm{sec})\end{array}$ & Nodes \\
\hline 1. & 0.39 & $<1$ & 2.01 & 1 & 1.42 & 1.07 & lim & 303 \\
2. & 0.36 & $<1$ & 2.16 & 1 & 0.97 & $<1$ & 1.58 & 1 \\
3. & 0.16 & $<1$ & 2.35 & 1 & 6.05 & 3.59 & lim & 75 \\
4. & 0.19 & $<1$ & 3.22 & 1 & 31.42 & 17.89 & lim & 17 \\
5. & 2.31 & $<1$ & 76.52 & 23 & 30.90 & 12.93 & lim & 5 \\
\hline
\end{tabular}

TABLE III: Various branch and bound algorithms applied on the modified test cases with timelim $=300 \mathrm{sec}$ and a relative gap of $1 \%$ (tol $=0.01$ ).

so for \# 2 only. Even though the rank condition is violated, the SDP relaxation continues to provide strong lower bounds as is indicated by the gap at the root node. We also try solving the problems to a tighter tolerance of $0.1 \%$ and the results are shown in Table IV.

\begin{tabular}{|c|c|c|c|c|c|c|c|c|}
\hline & \multicolumn{4}{|c|}{$\mathrm{SDP}-\mathrm{BB}$} & \multicolumn{4}{|c|}{ LD-BB } \\
\hline \# & $\begin{array}{r}\text { root } \\
\text { gap\% }\end{array}$ & $\begin{array}{l}\text { closed } \\
\text { gap\% }\end{array}$ & $\begin{array}{l}\text { Time } \\
(\mathrm{sec})\end{array}$ & Nodes & $\begin{array}{r}\text { root } \\
\text { gap\% }\end{array}$ & $\begin{array}{l}\text { closed } \\
\text { gap\% }\end{array}$ & $\begin{array}{l}\text { Time } \\
(\mathrm{sec})\end{array}$ & Nodes \\
\hline 1. & 0.39 & $<0.1$ & 11.01 & 31 & 1.42 & 1.07 & lim & 289 \\
\hline 2. & 0.36 & $<0.1$ & 37.12 & 91 & 0.87 & 0.85 & $\lim$ & 153 \\
\hline 3. & 0.16 & 0.12 & $\lim$ & 715 & 6.05 & 3.59 & $\lim$ & 75 \\
\hline 4. & 0.19 & $<0.1$ & 149.04 & 137 & 31.42 & 17.89 & $\lim$ & 17 \\
\hline 5. & 2.31 & 0.75 & $\lim$ & 110 & 30.90 & 12.93 & $\lim$ & 5 \\
\hline
\end{tabular}

TABLE IV: Various branch and bound algorithms applied on the modified test cases with timelim $=300 \mathrm{sec}$ and a relative gap of $1 \%(\operatorname{tol}=0.001)$.

For the tighter tolerance, the $\mathrm{SDP}-\mathrm{BB}$ algorithm took more nodes to close the gap to the given tolerance but the computational times are still within practical limits. There was however also no improvement in the best upper bound (globally optimal solution) on tightening the tolerance, indicating that a $1 \%$ tolerance would be sufficient in practice. The LD-BB algorithm was unable to certify the optimal solution to the tighter tolerance, and within the given time limit for all of the cases tested.

To study the difference in performance, we plot BestLB and BestUB for the 3 bus case (\#1). The global solution (BestuB) was found at the root node itself by the NLP solver, and the SDP based B \& B (solid line) had to explore 31 nodes in the search tree to certify the global optima to a $0.1 \%$ tolerance. The LD based approach (dash-dotted line) could not close the gap to $1 \%$, within the time limit.

The progress of the subgradient algorithm for the above

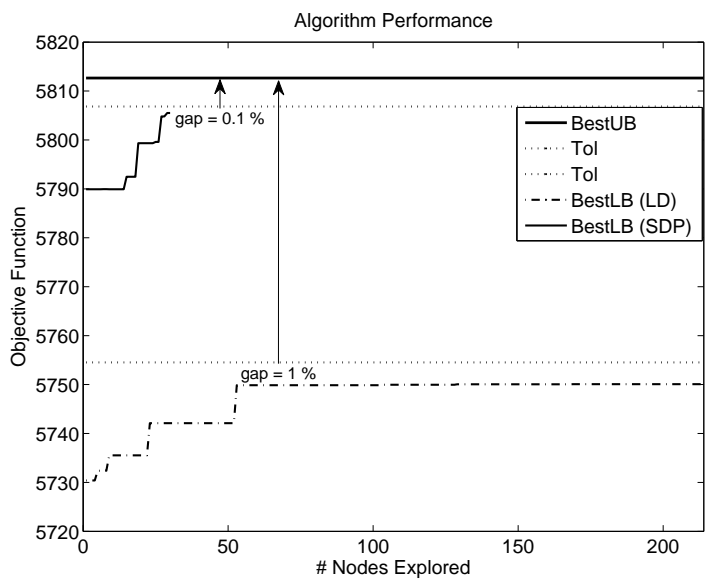

Fig. 3: Performance of the two B \& B algorithms on the 3 bus case.

case at the root node is plotted in Figure 4. The theoretical best value of the Lagrangian dual at the root node is given by the SDP solution at the root node (5789.91). The global solution is found by the NLP solver at the root node (5812.64). The optimal value of multipliers corresponding to this solution (optimal dual SDP vector) could not be found by the subgradient algorithm within 500 iterations. The best value (5730.38) was found in the iteration 449. The zigzag nature of the objective function is characteristic of the subgradient algorithm as it lacks an ascent property.

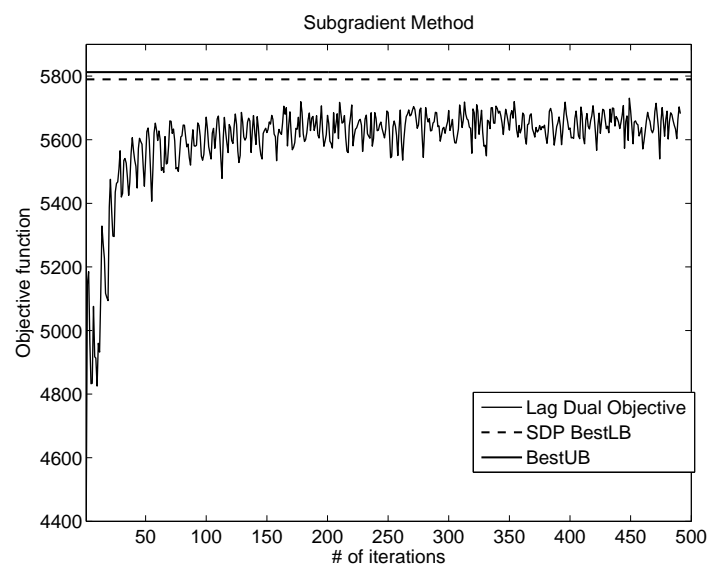

Fig. 4: Progress of the subgradient algorithm at the root node of the 3 bus case. 


\section{CONCLUSION}

In this work, we presented two branch and bound algorithms to solve the Optimal Power Flow problem to global optimality. The algorithms differ in their lower bounding approach, which is done either by solving the Lagrangian dual or the SDP relaxation of the OPF, both of which are theoretically equivalent but differ in computational performance. Both the algorithms were able to solve to global optimality all of the standard test cases at the root node itself indicating a zero duality gap. However when some of the parameters from the standard test cases were modified, a duality gap existed, and the SDP based B \& B algorithm was able to solve the cases tested to global optimality within a specified tolerance. The Lagrangian dual based algorithm was unable to close the gap due to computational difficulties in solving the non-smooth dual relaxation through a subgradient method. Alternate methods such as bundle methods could be used to overcome this. The performance of the algorithm on larger test cases also remains to be tested. We will also investigate the use of strengthening cuts in order to improve the performance of SDP-BB algorithm.

\section{APPENDIX}

Following Lavaei and Low [1] we introduce the matrices that are introduced in the definition of the OPF problem below. $\zeta_{i}$ denotes a vector of size $|\mathcal{N}|$ with a 1 at the $i$-th component and zeros elsewhere.

$$
\begin{aligned}
& Y_{\text {bus }, i}:=\zeta_{i} \zeta_{i}^{T} Y_{\text {bus }} \\
& Y_{\text {bus }, i j}:=y_{i j} \zeta_{i} \zeta_{i}^{T}-y_{i j} \zeta_{i} \zeta_{j}^{T} \\
& Y_{i}:=\frac{1}{2}\left[\begin{array}{ll}
\operatorname{Re}\left(Y_{\text {bus }, i}+Y_{\text {bus }, i}^{T}\right) & \operatorname{Im}\left(Y_{\text {bus }, i}^{T}-Y_{\text {bus }, i}\right) \\
\operatorname{Im}\left(Y_{\text {bus }, i}-Y_{\text {bus }, i}^{T}\right) & \operatorname{Re}\left(Y_{\text {bus }, i}+Y_{\text {bus }, i}^{T}\right)
\end{array}\right] \\
& \bar{Y}_{i}:=-\frac{1}{2}\left[\begin{array}{ll}
\operatorname{Im}\left(Y_{\text {bus }, i}+Y_{\text {bus }, i}\right) & \operatorname{Re}\left(Y_{\text {bus }, i}-Y_{\text {bus }, i}^{T}\right) \\
\operatorname{Re}\left(Y_{\text {bus }, i}^{T}-Y_{\text {bus }, i}\right) & \operatorname{Im}\left(Y_{\text {bus }, i}+Y_{\text {bus }, i}^{T}\right)
\end{array}\right] \\
& \left.Y_{i j}:=\frac{1}{2}\left[\begin{array}{cc}
\operatorname{Re}\left(Y_{\text {bus }, i j}+Y_{\text {bus }, i j}^{T}\right) & \operatorname{Im}\left(Y_{\text {bus }, i j}^{T}-Y_{\text {bus }, i j}\right) \\
\operatorname{Im}\left(Y_{\text {bus }, i j}-Y_{\text {bus }, i j}^{T}\right) & \operatorname{Re}\left(Y_{\text {bus }, i j}+Y_{\text {bus }, i j}^{T}\right)
\end{array}\right]\right) \\
& \bar{Y}_{i j}:=-\frac{1}{2}\left[\begin{array}{cc}
\operatorname{Im}\left(Y_{\text {bus }, i j}+Y_{\text {bus }, i j}^{T}\right) & \operatorname{Re}\left(Y_{\text {bus }, i j}-Y_{\text {bus }, i j}^{T}\right) \\
\operatorname{Re}\left(Y_{\text {bus }, i j}^{T}-Y_{\text {bus }, i j}\right) & \operatorname{Im}\left(Y_{\text {bus }, i j}+Y_{\text {bus }, i j}\right)
\end{array}\right] \\
& 0 \\
& M_{i}:=\left[\begin{array}{cc}
\zeta_{i} \zeta_{i}^{T} & 0 \\
0 & \zeta_{i} \zeta_{i}^{T}
\end{array}\right] \\
& M_{i j}:=\left[\begin{array}{cc}
\left(\zeta_{i}-\zeta_{j}\right)\left(\zeta_{i}-\zeta_{j}\right)^{T} & \left(\zeta_{i}-\zeta_{j}\right)\left(\zeta_{i}-\zeta_{j}\right)^{T}
\end{array}\right]
\end{aligned}
$$

\section{REFERENCES}

[1] J. Lavaei and S. H. Low, "Zero Duality Gap in Optimal Power Flow," IEEE Transactions on Power Systems, in press.

[2] B. Zhang and D. Tse, "Geometry of feasible injection region of power networks," http://arxiv.org/pdf/1107.1467.pdf, 2011.

[3] J. Lavaei, D. Tse, and B. Zhang, "Geometry of Power Flows in Tree Networks," in IEEE PES General Meeting, 2012.

[4] S. Bose, D. Gayme, S. Low, and K. M. Chandy, "Quadratically constrained quadratic programs on acyclic graphs with application to power flow," http://arxiv.org/pdf/1203.5599.pdf, 2012.

[5] S. Sojoudi and J. Lavaei, "Network topologies guaranteeing zero duality gap for optimal power flow problem," submitted for publication, 2011.

[6] J. Carpentier, "Contribution to the economic dispatch problem," Bulletin de la Societe Francoise des Electriciens, vol. 3, no. 8, pp. 431447, 1962, in French.

[7] B. Stott and O. Alşac, "Fast decoupled load flow," IEEE Trans. Power Apparatus and Systems, vol. PAS-93, no. 3, pp. 859-869, 1974.
[8] O. Alşac, J. Bright, M. Prais, and B. Stott, "Further developments in LP-based optimal power flow," IEEE Trans. Power Systems, vol. 5 , no. 3, pp. 699-711, 1990.

[9] K. Purchala, L. Meeus, D. Van Dommelen, and R. Belmans, "Usefulness of DC power flow for active power flow analysis," in IEEE PES General Meeting, 2005, pp. 2457-2462.

[10] B. Stott, J. Jardim, and O. Alşac, "DC Power Flow Revisited," IEEE Trans. Power Systems, vol. 24, no. 3, pp. 1290-1300, 2009.

[11] J. Momoh, M. El-Hawary, and R. Adapa, "A review of selected optimal power flow literature to 1993. Part I: Nonlinear and quadratic programming approaches," IEEE Transactions on Power Systems, vol. 14 , no. 1 , pp. 96-104, 1999.

[12] _ "A review of selected optimal power flow literature to 1993. Part II: Newton, linear programming and interior point methods," IEEE Transactions on Power Systems, vol. 14, no. 1, pp. 105-111, 1999.

[13] K. S. Pandya and S. K. Joshi, "A survey of optimal power flow methods," J. of Theoretical and Applied Information Technology, vol. 4, no. 5 , pp. $450-458,2008$.

[14] R. Jabr, "Radial distribution load flow using conic programming," IEEE Trans. Power Systems, vol. 21, no. 3, pp. 1458-1459, 2008.

[15] _ - "A conic quadratic format for the load flow equations of meshed networks," IEEE Trans. Power Systems, vol. 22, no. 4, pp. 2285-2286, 2007.

[16] — "Optimal power flow using an extended conic quadratic formulation," IEEE Trans. Power Systems, vol. 23, no. 3, pp. 1000-1008, 2008.

[17] X. Bai, H. Wei, K. Fujisawa, and Y. Wang, "Semidefinite programming for optimal power flow problems," Intl J. of Electrical Power \& Energy Systems, 2008

[18] X. Bai and H. Wei, "Semidefinite programming-based method for security-constrained unit commitment with operational and optimal power flow constraints," Generation, Transmission \& Distribution, 2009.

[19] D. Gayme and U. Topcu, "Optimal power flow with distributed energy storage dynamics," in American Control Conference, 2011.

[20] B. Lesieutre, D. Molzahn, A. Borden, and C. L. DeMarco, "Examining the limits of the application of semidefinite programming to power flow problems," in $49^{t h}$ Annual Allerton Conference on Communications, Control and Computing, 2011, pp. 1492-1499.

[21] Y. V. Makarov, Z. Dong, Y., and J. Hill, D., "On convexity of power flow feasibility boundary," IEEE Trans on Power Systems, vol. 23, no. 2, pp. 811-813, 2008.

[22] M. Farivar and S. H. Low, "Branch Flow Model: Relaxations and Convexification," http://arxiv.org/abs/1204.4865, 2012.

[23] D. T. Phan, "Lagrangian Duality and Branch-and-Bound Algorithms for Optimal Power Flow," Operations Research, accepted.

[24] A. d'Aspremont and S. Boyd, "Relaxations and Randomized Methods for Nonconvex QCQPs," Lecture notes of EE392o, Stanford University, Autumn 2003.

[25] S. Boyd and A. Mutapcic, "Subgradient Methods," Lecture notes of EE364b, Stanford University, Winter 2006-07.

[26] S. Boyd and L. Vandenberghe, Convex Optimization. Cambridge University Press, 2004.

[27] M. Tawarmalani and N. Sahinidis, Convexification and Global Optimization in Continuous and Mixed-Integer Nonlinear Programming: Theory, Algorithms, Software, and Applications, ser. Nonconvex Optimization and Its Applications. Kluwer Academic Publishers, 2002.

[28] A. Drud, "CONOPT - A large scale GRG code," ORSA J. Computing, vol. 6, pp. 207-218, 1994.

[29] P. Camerini, L. Fratta, and F. Maffioli, "On improving relaxation methods by modifying gradient techniques." Math. Programming Study, vol. 3, pp. 26-34, 1975.

[30] J. Löfberg, "Yalmip : A toolbox for modeling and optimization in MATLAB," in Proceedings of the CACSD Conference, Taipei, Taiwan, 2004. [Online]. Available: http://control.ee.ethz.ch/ $\sim \sim$ joloef/ yalmip.php

[31] "Power World," http://www.powerworld.com.

[32] R. Zimmerman, C. Murillo-Sanchez, and R. J. Thomas, "MATPOWER Steady-State Operations, Planning and Analysis Tools for Power Systems Research and Education," IEEE Transactions on Power Systems, vol. 26, pp. 12-19, Feb 2011

[33] M. Tawarmalani and N. V. Sahinidis, "A polyhedral branch-and-cut approach to global optimization," Mathematical Programming, vol. 103, pp. 225-249, 2005.

[34] A. Brooke, D. Kendrick, and A. Meeraus, GAMS: A User's Guide. South San Francisco: The Scientific Press, 1988. 